\title{
Local structure of silver clusters in the channels of zeolite $4 \mathrm{~A}$
}

\section{T. Miyanaga, H. Hoshino and H. Endo}




\section{Local structure of silver clusters in the channels of zeolite $4 \mathrm{~A}$}

\author{
T. Miyanaga*, H. Hoshino ${ }^{a}$ and $H$. Endo ${ }^{b}$ \\ Faculty of Science and Technology, Hirosaki University, \\ Hirosaki, 036-8561 Japan \\ ${ }^{a}$ Faculty of Education, Hirosaki University, Hirosaki, 036-8560 \\ Japan \\ ${ }^{b}$ Faculty of Engineering, Fukui Institute of Technology, Fukui, \\ 910-8505 Japan \\ ^E-mail: takaf@cc.hirosaki-u.ac.jp
}

The Ag $K$-edge EXAFS measurements have been carried out at $50 \mathrm{~K}$ for fully $\mathrm{Ag}^{+}$-exchanged zeolite $4 \mathrm{~A}(\mathrm{Ag}-4 \mathrm{~A})$ dehydrated at different temperatures under vacuum. In the Fourier transforms of the EXAFS oscillation the two distinct peaks appear around $2.2 \AA$ and $3.0 \AA$, and a shoulder around $2.8 \AA$. The structural parameters (distance, coordination number and root mean square displacement) were derived by a three-shell (two $\mathrm{Ag}-\mathrm{O}$ contacts and $\mathrm{Ag}-\mathrm{Ag}$ contact) curve-fitting analysis. It is found that a silver cluster is formed with $\mathrm{Ag}-\mathrm{Ag}$ distance $2.82 \pm 0.02 \AA$ and coordination number 4.9 \pm 0.2 . When the silver cluster is formed $6 \mathrm{Ag}^{0}$ atoms and $8 \mathrm{Ag}^{+}$ ions are arranged in a cubic closed-packed manner in the cage of the Ag-4A zeolite. The $8 \mathrm{Ag}^{+}$ions and $6 \mathrm{Ag}^{0}$ atoms are confined in the framework of oxygens at distances of $2.28 \pm 0.02$ and $2.88 \pm 0.02 \AA$, respectively.

\section{Keywords: EXAFS at $50 \mathrm{~K}, \mathrm{Ag}$ cluster, zeolite 4A}

\section{Introduction}

In the fully $\mathrm{Ag}^{+}$-exchanged zeolite $4 \mathrm{~A}(\mathrm{Ag}-4 \mathrm{~A})$ the $12 \mathrm{Ag}^{+}$ions are present inside the zeolite cages, as needed to balance the anionic charge of the zeolite framework. The white color of the hydrated Ag-4A changes through yellow to brown by heating under vacuum. These changes in color by heating under vacuum are related to the formation of silver clusters due to the interaction of the silver atoms produced by autoreduction with the other silver atoms or $\mathrm{Ag}^{+}$ions obtained by dehydration (Sun \& Seff, 1994). The presence of silver clusters has been found by X-ray diffraction experiments of the zeolite single crystal (Kim \& Seff, 1987, Gellens et al.,1981). The EXAFS technique provides important structural information of the changes in the local environment around a central atom in disordered systems. In the previous paper we have reported the EXAFS experiment for the vacuum-dehydrated Ag-4A powder at $298 \mathrm{~K}$ (Miyanaga et al., 1999) and suggested the presence of a silver cluster located in the zeolite cage. It is interesting to carry out the EXAFS measurement at much lower temperature in order to reduce the thermal effect. In this paper, we report the results of the EXAFS measurements on the $\mathrm{Ag} K$-edge for the vacuum-dehydrated fully exchanged $\mathrm{Ag}-4 \mathrm{~A}$ powder at $50 \mathrm{~K}$.

\section{Experimental}

The fully $\mathrm{Ag}^{+}$-exchanged 4A (Ag-4A) powder samples were prepared by immersing $\mathrm{Na}-4 \mathrm{~A}\left(\mathrm{Na}_{12}\left[\left(\mathrm{AlO}_{2}\right)_{12}\left(\mathrm{SiO}_{2}\right)_{12}\right] 27.5 \mathrm{H}_{2} \mathrm{O}\right)$ zeolite in an aqueous $\mathrm{AgNO}_{3}$ solution at $25^{\circ} \mathrm{C}$. The details of the sample preparation are described elsewhere (Hoshino, 1996, Miyanaga et al. 1999). The white Ag-4A powder (fully hydrated) was obtained by drying in air for 5 days at $25^{\circ} \mathrm{C}$. We call this sample Ag-4A-25. The following three samples were prepared by heating Ag-4A-25 under vacuum of $10^{-3}$ Torr for $10 \mathrm{~h}$ at different temperatures: Ag-4A-200 (dehydrated at $200^{\circ} \mathrm{C}$, yellow), Ag- $4 \mathrm{~A}-$
350 (dehydrated at $350^{\circ} \mathrm{C}$, brown) and $\mathrm{Ag}-4 \mathrm{~A}-500$ (dehydrated at $500^{\circ} \mathrm{C}$, pale brown). Each sample was pasted on an adhesive tape in a glove box filled by $\mathrm{He}$ gas. The tape was mounted on a copper holder set in a chamber where evacuation up to $10^{-5}$ Torr and cooling down to $50 \mathrm{~K}$ are possible. $\mathrm{Ag} K$-edge EXAFS spectra were measured at beamline BL-10B of the Photon Factory in KEK. A $\mathrm{Si}(311)$ channel-cut monochromator was used, and energy and current of the storage ring were $3.0 \mathrm{GeV}$ and $250 \sim 400 \mathrm{~mA}$, respectively. EXAFS spectra were recorded in transmission mode using ionization chamber detectors. The EXAFS interference function extracted from the absorption spectra was Fourier transformed by XANADU program (Sakane et al., 1993). In order to obtain the structural parameters, the EXAFS function was fitted by a non-linear least-squares method. In the fitting process, theoretical parameters calculated by the FEFF6 code (Rehr et al. 1991) were used. The $k$-range for the curve-fitting method was 3.5 11.5 $\AA^{-1}$. The energy correction was made by fitting our results on $\mathrm{Ag}$ foil and $\mathrm{AgNO}_{3}$ as standard samples.

\section{Results and Discussion}

Figure 1 shows the EXAFS spectra, $k \chi(k)$, measured at the $\mathrm{Ag} K$ edge for Ag-4A-25, Ag-4A-200, Ag-4A-350 and Ag-4A-500. The oscillations are detectable in the $k$ region up to $12 \AA^{-1}$. For Ag-4A200 there appears a shoulder in the $k$ region between 2 and $3 \AA^{-1}$. The spectrum for white Ag-4A-25 is considerably different from those for colored Ag-4A in the $k$ region between 3 and $8 \AA^{-1}$.

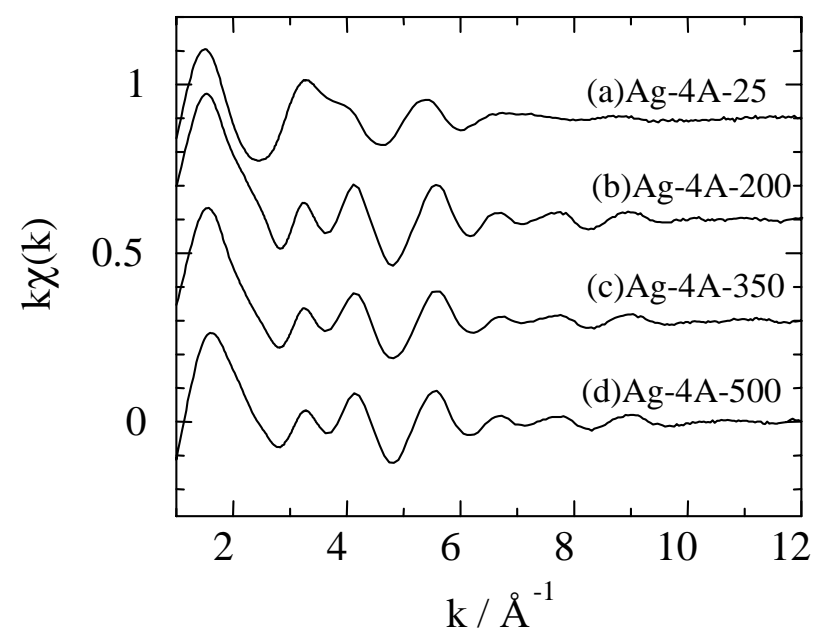

Figure 1.

Ag $K$-edge EXAFS, $k \chi(k)$, functions for four different $\mathrm{Ag}-4 \mathrm{~A}$ zeolites. (a) Ag-4A-25 (fully hydrated, dried at $25^{\circ} \mathrm{C}$, measured at $298 \mathrm{~K}$ )

(b) Ag-4A-200 (dehydrated at $200^{\circ} \mathrm{C}$, measured at $50 \mathrm{~K}$ ),

(c)Ag-4A-350 (dehydrated at $350^{\circ} \mathrm{C}$, measured at $50 \mathrm{~K}$ ) and

(d)Ag-4A-500 (dehydrated at $500^{\circ} \mathrm{C}$, measured at $50 \mathrm{~K}$ ).

Figure 2 shows the Fourier transforms $\mathrm{F}(r)$ of EXAFS spectra for three different Ag-4A samples measured at $50 \mathrm{~K}$ and for hydrated Ag-4A-25 measured at 298K. There are two dominant peaks in the $\mathrm{F}(r)$. In the previous paper (Miyanaga et al. 1999), we suggested that the first peak around $2.2 \AA$ corresponds to the Ag-O contact and the second one around $3.0 \AA$ to a $\mathrm{Ag}-\mathrm{Ag}$ contact. As the vacuumdehydration temperature of $\mathrm{Ag}-4 \mathrm{~A}$ zeolite increases, the peak around $3.0 \AA$ becomes prominent. A shoulder appears around $2.8 \AA$ for the vacuum-dehydrated $\mathrm{Ag}-4 \mathrm{~A}$ samples at $50 \mathrm{~K}$ where the thermal 
excitation is substantially reduced, though it was not clearly observed at $298 \mathrm{~K}$ (Miyanaga et al. 1999). The shoulder is considered to be caused by the presence of another Ag-Ag or Ag-O contact.

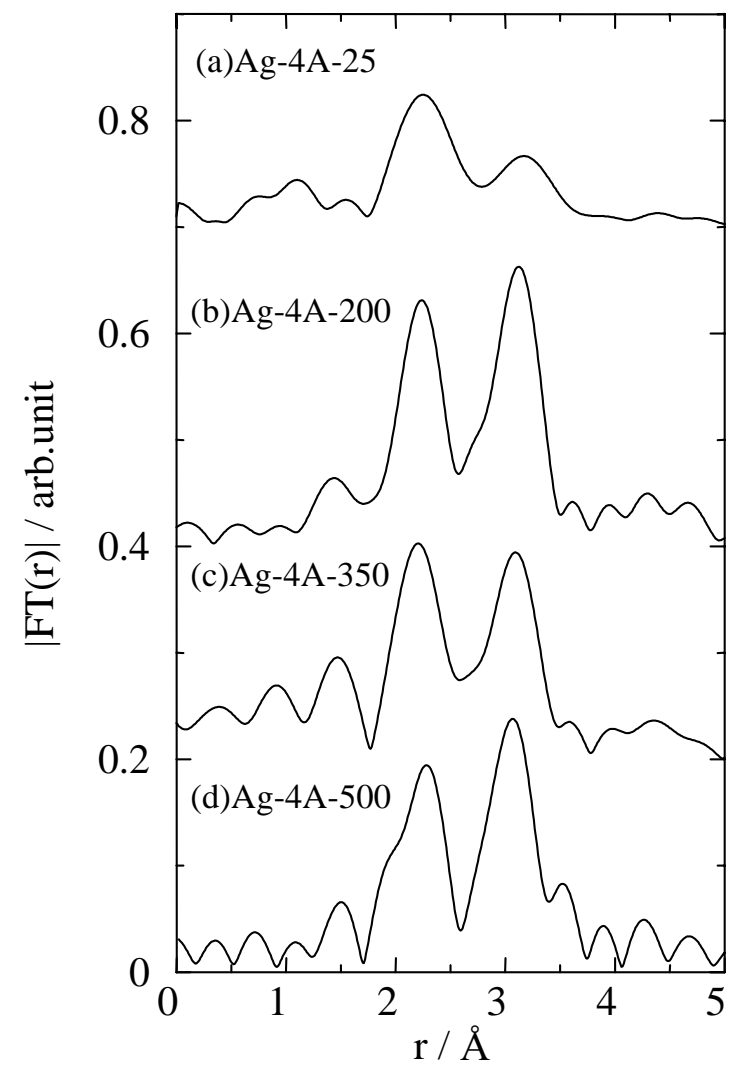

Figure 2.

Fourier transforms of EXAFS, F(r), for four different Ag-4A zeolites. (a) Ag4A-25, (b)Ag-4A-200, (c)Ag-4A-350 and (d)Ag-4A-500.

In order to derive the structural parameters, three-shell curvefitting analyses were carried out. The three-shell model consists of two Ag-O (long and short contact distances) and one Ag-Ag contact. Figure 3 shows the comparison for Ag-4A-500 at $50 \mathrm{~K}$ between the experimental and calculated $k \chi(k)$ curves by using the structural parameters. The agreement is fairly good. Although we have tried curve-fitting analysis by using another three-shell model consisting of one Ag-O and two Ag-Ag contacts (short and long), it is found that quite unreasonable values are obtained for some structural parameters and the fitting in $k$ range between 3.5 and $6.5 \AA^{-1}$ was not better than that shown in Figure 3. In Table 1 the structural parameters are compiled for hydrated Ag-4A-25 measured at $298 \mathrm{~K}$, and those for Ag-4A-200, Ag-4A-350 and Ag-4A-500 measured at $50 \mathrm{~K}$.

In the hydrated $\mathrm{Ag}-4 \mathrm{~A}-25$ there are $\mathrm{Ag}^{+}$ions. The short distance between $\mathrm{Ag}^{+}$and $\mathrm{O}$ is $2.38 \pm 0.02 \AA$ and the coordination number of $\mathrm{O}$ around a central $\mathrm{Ag}^{+}$is $6.0 \pm 0.2$ (Table 1). These $\mathrm{Ag}^{+}$ions are considered to be in contact with framework oxygens (Gellens et al., 1981, Kim and Seff, 1987). The long $\mathrm{Ag}^{+}$-O distance, $2.84 \pm 0.02 \AA$ with the coordination number of $\mathrm{O}$ around a central $\mathrm{Ag}^{+}$of $2.2 \pm 0.2$ corresponds to another $\mathrm{Ag}^{+}$- framework $\mathrm{O}$. The $\mathrm{Ag}-\mathrm{Ag}$ distance,

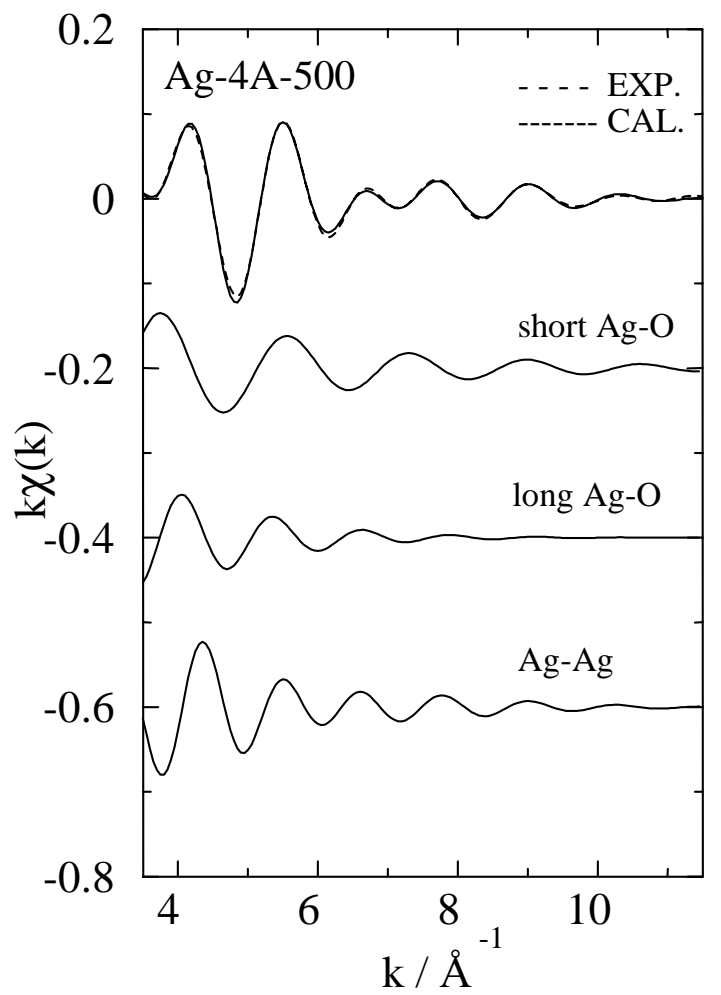

Figure 3.

Comparison for the Ag-4A-500 measured at $50 \mathrm{~K}$ between the experimental curve $k \chi(k)$ and the calculated curve by using the structural parameters. Individual contributions due to short $\mathrm{Ag}-\mathrm{O}$, long $\mathrm{Ag}-\mathrm{O}$ and $\mathrm{Ag}-\mathrm{Ag}$ are shown together.

$2.88 \pm 0.02 \AA$, corresponds to $\mathrm{a}^{\mathrm{Ag}} \mathrm{g}^{+} \mathrm{Ag}^{+}$contact. This distance is longer than the sum of the ionic radii of two $\mathrm{Ag}^{+}$ions, $2.54 \AA$, due to the Coulomb repulsion between $\mathrm{Ag}^{+}$ions in the zeolite cage. The coordination number of $\mathrm{Ag}^{+}$around a central $\mathrm{Ag}^{+}$is $3.0 \pm 0.2$. This suggests that $8 \mathrm{Ag}^{+}$ions form a cube and 4 other $\mathrm{Ag}^{+}$ions are distributed at the face-centered positions of the cube in the cage of. the hydrated Ag-4A zeolite

As the vacuum-dehydration of Ag-4A-25 proceeds, the amount of water decreases and $\mathrm{Ag}^{+}$ions are reduced to $\mathrm{Ag}$ atoms $\left(\mathrm{Ag}^{0}\right)$, which gives rise to changes in color of $\mathrm{Ag}-4 \mathrm{~A}$. It is found that the short Ag-O distance, $2.28 \pm 0.02 \AA$ and the coordination number of $\mathrm{O}$ around a central $\mathrm{Ag}$ of $3.0 \pm 0.2$ are independent of the dehydration temperature within experimental errors (Table 1). By comparing with the results of the hydrated Ag-4A-25 the short Ag-O distance in the vacuum-dehydrated $\mathrm{Ag}-4 \mathrm{~A}$ is considered to correspond to the $\mathrm{Ag}^{+}$-framework $\mathrm{O}$ contact. The long $\mathrm{Ag}-\mathrm{O}$ distance changes from $2.88 \pm 0.02$ to $2.91 \pm 0.02 \AA$ with increasing dehydration temperature, while the coordination number of $\mathrm{O}$ around a central $\mathrm{Ag}, 3.0 \pm 0.02$, is almost constant. The long $\mathrm{Ag}-\mathrm{O}$ distance for vacuum-dehydrated Ag-4A elongates by dehydration, while the coordination number is almost constant. This arises from a weakening of the Coulomb interaction between $\mathrm{Ag}$ and framework $\mathrm{O}$ as $\mathrm{Ag}^{+}$ions are reduced to $\mathrm{Ag}^{0}$ atoms. It is concluded that the long $\mathrm{Ag}-\mathrm{O}$ distance corresponds to the $\mathrm{Ag}^{0}$ - framework $\mathrm{O}$ contact for the completely dehydrated $\mathrm{Ag}$ $4 \mathrm{~A}-500$. 
molecules and clusters

Table 1 Structural parameters ( $r$ : contact distance, $N$ : coordination number and $\sigma$ : root mean square displacement) for fully hydrated Ag-4A zeolites.

\begin{tabular}{|c|c|c|c|c|c|c|c|c|c|}
\hline & \multicolumn{3}{|c|}{ short Ag-O } & \multicolumn{3}{|c|}{ long Ag-O } & \multicolumn{3}{|c|}{$\mathrm{Ag}-\mathrm{Ag}$} \\
\hline & $r(\AA)$ & $N$ & $\sigma(\AA)$ & $r(\AA)$ & $N$ & $\sigma(\AA)$ & $r(\AA)$ & $N$ & $\sigma(\AA)$ \\
\hline $\begin{array}{c}\text { Ag-4A-25 } \\
(298 \mathrm{~K})\end{array}$ & 2.38 & 6.0 & 0.14 & 2.84 & 2.2 & 0.13 & 2.88 & 3.0 & 0.14 \\
\hline $\begin{array}{c}A g-4 A-200 \\
(50 \mathrm{~K})\end{array}$ & 2.28 & 3.0 & 0.10 & 2.88 & 3.0 & 0.12 & 2.83 & 5.6 & 0.13 \\
\hline $\begin{array}{c}A g-4 A-350 \\
(50 \mathrm{~K})\end{array}$ & 2.27 & 2.8 & 0.10 & 2.87 & 3.0 & 0.12 & 2.82 & 4.0 & 0.13 \\
\hline $\begin{array}{c}A g-4 A-500 \\
(50 \mathrm{~K})\end{array}$ & 2.28 & 3.1 & 0.10 & 2.91 & 2.9 & 0.12 & 2.81 & 4.9 & 0.13 \\
\hline
\end{tabular}

For Ag-4A-200 and Ag-4A-350 the $\mathrm{Ag}^{+}-\mathrm{O}$ contact is considered to be present together with the $\mathrm{Ag}^{0}-\mathrm{O}$ contact. The $\mathrm{Ag}-\mathrm{Ag}$ distance decreases from $2.83 \pm 0.02$ to $2.81 \pm 0.02 \AA$ with increasing dehydration temperature. The $\mathrm{Ag}-\mathrm{Ag}$ contact distance in the vacuum-dehydrated Ag-4A is shorter than that in the hydrated one, because the Coulomb repulsion between $\mathrm{Ag}^{+}$ ions becomes weaker by producing $\mathrm{Ag}^{0}$. It is difficult to distinguish the $\mathrm{Ag}^{+}-\mathrm{Ag}^{0}$ contact from the $\mathrm{Ag}^{0}-\mathrm{Ag}^{0}$ contact in the curve-fitting analysis of the EXAFS oscillation, which results in a relatively large root mean square displacement, $0.13 \pm 0.3 \AA$. The coexistence of $\mathrm{Ag}^{+}$ions and $\mathrm{Ag}^{0}$ atoms is associated with the formation process of silver clusters. $6 \mathrm{Ag}^{0}$ atoms are on the face-centered positions of the cube (unit length $3.97 \AA$ ) formed by $8 \mathrm{Ag}^{+}$ions in the zeolite cage. For this arrangement the coordination number of $\mathrm{Ag}$ around a central $\mathrm{Ag}$ is estimated to be 5.1 which is in agreement with the experimental value 4.9 \pm 0.2 for Ag-4A-500.

\section{Conclusion}

EXAFS measurements of Ag $K$-edge were carried out on the vacuum-dehydrated powdered $\mathrm{Ag}-4 \mathrm{~A}$ zeolite at $50 \mathrm{~K}$. In the Fourier transforms $\mathrm{F}(r)$ of EXAFS spectra there appear two distinct peaks around $2.2 \AA$ and $3.0 \AA$, respectively and a shoulder around $2.8 \AA$. The EXAFS data have been analyzed by the three-shell curve-fitting method. It is found that a silver cluster is formed with $\mathrm{Ag}-\mathrm{Ag}$ contact distance $2.82 \pm 0.02 \AA$ with the coordination number $4.9 \pm 0.2$. For the formation of the silver cluster $6 \mathrm{Ag}^{0}$ atoms and $8 \mathrm{Ag}^{+}$ions are arranged in a cubic closed-packed manner in the cage of the Ag-4A zeolite. $8 \mathrm{Ag}^{+}$ ions and $6 \mathrm{Ag}^{0}$ atoms are in contact with the framework oxygens with distances $2.28 \pm 0.02 \AA$ and $2.88 \pm 0.02 \AA$, respectively.

\section{Acknowledgements}

We thank Dr. H. Ikemoto and Mr. M. Yuza for their support on the EXAFS experiments.

\section{References}

Gellens, L.R., Mortier, W.J. and Uytterhoeven, J.B. (1981), Zeolites, 1, 11-18.

Hoshino, H., (1996) Bulletin of the Faculty of Education, Hirosaki University, 75, 29-37 .

Kim, Y. and Seff, K., (1987), J. Phys. Chem. 91(3), 668-674.

Miyanaga, T., Hoshino, H., Endo, H. and Sakane, H., (1999), J. Synchrotron Rad. 8, 442-444.

Rehr, J.J., Mustre de Leon, J., Zabinsky, S.I. and Albers, R.C., (1991) J. Am. Chem. Soc. 113, 5135-5140.

Sakane, H., Miyanaga, T., Watanabe, I., Matsubayashi, N., Ikeda, S. and Yokoyama, Y., (1993), Jpn. J. Appl. Phys., 32, 4641-4647.

Sun T. and Seff K., (1994), Chem. Rev. 94(4), 857-870. 